\title{
Depression among older adults in the United States and England
}

\author{
Kara Zivin, $\mathbf{P h D}^{1,2}$, David J. Llewellyn, $\mathrm{PhD}^{5,6}$, lain A. Lang, $\mathrm{PhD}^{4}$, Sandeep Vijan, MD, \\ MS $^{3,7}$, Mohammed U. Kabeto, $\mathbf{M S}^{3}$, Erin M. Miller, $\mathbf{M S}^{2}$, and Kenneth M. Langa, MD, PhD ${ }^{3,7,8}$ \\ ${ }^{1}$ Department of Veterans Affairs, Health Services Research and Development (HSR\&D) Center of \\ Excellence, Serious Mental Illness Treatment Research and Evaluation Center (SMITREC), Ann \\ Arbor, MI \\ ${ }^{2}$ Department of Psychiatry, University of Michigan Medical School, Ann Arbor, MI \\ ${ }^{3}$ Division of General Medicine, Department of Medicine, University of Michigan Medical School, \\ Ann Arbor, MI \\ ${ }^{4}$ Epidemiology and Public Health Group, Peninsula Medical School, Exeter, UK \\ ${ }^{5}$ Department of Neuropsychology, Addenbrooke's Hospital University of Cambridge, Cambridge, \\ UK \\ ${ }^{6}$ Department of Public Health and Primary Care, University of Cambridge, Cambridge, UK \\ ${ }^{7}$ Department of Veterans Affairs, Health Services Research and Development (HSR\&D) Center of \\ Excellence, Center for Practice Management and Outcomes Research Ann Arbor, MI \\ ${ }^{8}$ Institute for Social Research, University of Michigan, Ann Arbor, MI
}

\section{Abstract}

Context-Depression negatively affects health and well-being among older adults, but there have been no nationally representative comparisons of depression prevalence among older adults in England and the United States.

Objective-We sought to compare depressive symptoms among older adults in these countries and identify sociodemographic and clinical correlates of depression in these countries.

Design and Setting-We assessed depressive symptoms in non-Hispanic whites aged 65 and over in 2002 in two nationally representative, population-based studies: the US Health and Retirement Study (HRS) and English Longitudinal Study of Ageing (ELSA).

Participants-8,295 HRS respondents and 5,208 ELSA respondents.

Main Outcome Measures-We measured depressive symptoms using the eight-item Center for Epidemiologic Studies Depression Scale (CES-D). We determined whether depressive symptom differences between the US and England were associated with sociodemographic characteristics, chronic health conditions, and health behaviors.

Results-Significant depressive symptoms (CES-D score $\geq 4$ ) were more prevalent in English than US adults $(17.6 \%$ vs. $14.6 \%$, adjusted Wald test $\mathrm{F}(1,1593)=11.4, \mathrm{p}<0.001)$. Adjusted rates

Corresponding author: Kara Zivin, PhD, University of Michigan Medical School, Department of Psychiatry, Rachel Upjohn Building, 4250 Plymouth Road, Box 5765, Ann Arbor, MI 48109. kzivin@umich.edu; Phone: 734-769-7100 x56009; fax 734-845-3249.

The authors have no conflicts of interest to report.

Declaration of interest: The organizations funding this study had no role in the design or conduct of the study; in the collection, management, analysis, or interpretation of the data; or in the preparation, review, or approval of the manuscript. 
of depressive symptoms in England were 19\% higher compared to the US (OR: 1.19, 95\% CI: $1.01,1.40)$. US adults had higher levels of education, and net worth, but lower levels of ADL/ IADL impairments, tobacco use, and cognitive impairment, which may have contributed to relatively lower levels of depressive symptoms in the US.

Conclusions-Older adults in the US had lower rates of depressive symptoms than their English counterparts despite having more chronic health conditions. Future cross-national studies should identify how depression treatment influences outcomes in these populations.

\section{Keywords}

depression; Health and Retirement Study English Longitudinal Study of Ageing; older adults

\section{Introduction}

Depression among older adults is a significant and growing public health problem worldwide.(1) Depression is not part of normal aging, but it is associated with increased medical burden, health service utilization, longer hospital stays, disability, and more functional impairment than most medical disorders. Depression is also associated with low socioeconomic status, lack of social support or contact, bereavement, mortality and suicide in later life. Inadequate identification and treatment of depression is an important problem for older adults due to depression's negative impact on patients and their families.(2) However, effective treatments for depression in this population are available. Treatment for depression may improve physical functioning and depression outcomes in older adults. Attributing depression to old age may be a barrier to treatment seeking, and there are many potential reasons for poor treatment adherence in older adults, including stigma, negative patient beliefs about treatment, physical and psychiatric comorbidity, and costs of care.(3)

Research has identified factors in the United States and England that may contribute to depression in older adults. The United States has higher levels of many medical disorders (e.g. cerebrovascular diseases, cancer), which are also associated with depression.(4) In England, older adults report lower levels of self-rated health, and also have lower income and education levels, which are all associated with depression.(5) While there have been a variety of studies that have examined rates of depression in older adults either in England(6) or the United States,(7) there is little literature comparing rates across these two countries. (8) Although there is some precedent for cross-national comparisons of health disorders using surveys, existing studies do not capture rates and correlates of depression in both countries using similar populations and identical measures.

Therefore, we sought to compare the prevalence of depressive symptoms in the United States and England using the same depression measures administered to nationally representative samples of older adults in both countries. Our goals were to assess: 1) whether there were differences in depressive symptoms in adults aged 65 and older in these two countries, and 2) which socioeconomic and health measures account for differences in depressive symptoms across the two countries. Identifying characteristics in each country associated with increased risks of depression in older adults could assist in the development of targeted interventions.

\section{Methods}

\section{Study populations}

We used data on adults aged 65+ from the 2002 waves of the US Health and Retirement Study (HRS)(9) and the English Longitudinal Study of Ageing (ELSA).(10) Both studies are 
longitudinal, nationally representative studies administered biannually to adults aged $51+$ in the United States and 50+ in England. The studies were developed collaboratively and were designed to have considerable question overlap to facilitate cross-national comparisons of health and wealth associated with aging.

For this study, we included individuals who were aged 65+. To maximize the comparability of the US and English samples, we included respondents of white race only since ELSA contains very few $(\mathrm{n}=156)$ non-white individuals. We excluded individuals represented by a proxy (1,171 [12\%] in the HRS and 96 [2\%] in the ELSA) since the depression questions were not administered to those respondents. The overall response rate among eligible respondents was $87 \%$ for the HRS and $67 \%$ for ELSA.

The HRS survey was administered by telephone ( $71 \%$ of the sample) and in-person ( $29 \%$ of the sample). The HRS attempts to interview older respondents (age > 80) in-person whenever possible. All ELSA interviews were performed in-person.

This study was approved by the Institutional Review Board at the University of Michigan Medical School. All study participants provided informed written consent. Respondent anonymity was maintained because data used for our analyses did not contain any unique identifying information.

\section{Study measures}

Depression-Both the HRS and ELSA used the eight item version of the Center for Epidemiologic Studies Depression Scale (CES-D) to measure depressive symptoms.(11) The CESD has been widely used in studies of late life depression, and has good psychometric properties for use in these populations. To determine depressive symptoms, each self-respondent was asked the following questions with response options of 'yes' or 'no': 1) Much of the time during the past week, I felt depressed; 2) I felt everything I did was an effort; 3) My sleep was restless; 4) I was happy; 5) I felt lonely; 6) I enjoyed life; 7) I felt sad; 8) I could not "get going". For each respondent, the total number of "yes" responses to questions $1,2,3,5,7,8$, and the "no" responses to questions 4 and 6 were summed to arrive at a total depressive symptom score ranging from 0 to 8 . If an individual was missing two or more of the eight items, he/she was excluded from the analyses. As a result, we excluded $4(0.05 \%)$ and $68(1.3 \%)$ individuals from the HRS and ELSA, respectively, due to nonresponse. Our overall sample was 8,295 HRS respondents and 5,208 from ELSA. We classified those who reported four or more depressive symptoms as having significant depressive symptoms, a cut-off that has been found to produce comparable results to the 16symptom cut-off for the well-validated 20-item CES-D scale.(12) This was our outcome variable to investigate the differences in significant depressive symptoms in the US and England.

Sociodemographic characteristics-We included age (65-74, 75-84, 285), gender, household net worth (tertiles; 2002 US dollars), and level of education as sociodemographic measures and independent variables in the analyses. British pounds were converted to 2002 US dollars using the average currency exchange rate for 2002. Measures of education differ in the HRS and ELSA. Following Banks and colleagues,(4) we identified low, middle, and high education categories for each country ( 0 to 12 years of school, 13 to 15 years, and $>16$ years, respectively, in the US; qualified to lower than "Ordinary-level" [O-level], O-level to lower than "Advanced-level" [A-level], and A-level or higher, respectively, in England).

Health conditions and treatments-Participants in the HRS and ELSA were asked about the presence of common chronic health conditions using the question, "Has a doctor ever told you that you had...?" For this analysis, we included stroke, diabetes, heart disease, 
hypertension, lung disease, and cancer. Smoking status (never, former, or current), and alcohol consumption (average number of days/week that alcohol was consumed over the last 3 months [HRS] or 12 months [ELSA]) were assessed similarly in both the HRS and ELSA.

Both the HRS and ELSA assessed cognitive function using tests of immediate and delayed recall of 10 common nouns, and orientation to day, date, month, and year. These three tests resulted in a cognitive scale ranging from 0 to 24 possible points (10 points for immediate recall, 10 points for delayed recall, and 4 points for orientation). If a respondent refused to provide an answer for a cognitive item, they were assigned a score of zero for that item. Ninety-five (1\%) HRS respondents and 43 (1\%) ELSA respondents refused to answer for one or more of the cognitive items.

The HRS and ELSA determine the presence of limitations in independent function by asking about difficulty with 6 activities of daily living (ADLs; eating, getting in and out of bed, toileting, dressing, bathing, walking across a room) and 5 instrumental activities of daily living (IADLs; preparing meals, grocery shopping, making phone calls, taking medications, managing money).

\section{Analysis Plan}

We calculated means and frequencies of each independent variable in each country using ttests and Pearson chi-square tests. We pooled data from the US and England and estimated a logistic regression model with the dichotomous depression indicator (0-3 vs. 4+ CES-D symptoms) as the dependent variable. Using a dichotomous predictor variable indicating the country ( 0 for the US; 1 for England) to determine cross-national differences in depressive symptoms, the adjusted logistic regression analysis also accounted for sociodemographic and health variables.

All analyses were performed using STATA 9.1 and used the HRS or ELSA sample weights to adjust for survey non-response and for the complex sampling design (stratification and clustering) of each study.

\section{Results}

The characteristics of the US and English samples are shown in Table 1. There was a slightly higher proportion of women in the US sample. Educational attainment and average household net worth (in 2002 US dollars) were significantly higher in the US. The English sample had significantly lower levels of chronic disease (e.g. physical health condition) for every condition included in the analysis. However, despite lower levels of chronic disease, English adults reported more limitations in ADLs/IADLs, and more cognitive impairment. English adults were more likely to be current smokers and drank alcohol on more days per week than those in the US.

Significant depressive symptoms by country are also shown in Table 1. Of the 8,295 HRS respondents, $1245(14.6 \%)$ had 4 or more depressive symptoms, whereas of the 5,208 ELSA respondents, $906(17.6 \%)$ had 4 or more symptoms. Furthermore, 56.4\% of the US sample had any depressive symptoms whereas $63.5 \%$ of the English sample did. Differences by individual CES-D items indicate that the US had significantly lower levels of feeling depressed, feeling like everything was an effort, and sleepiness, and higher levels of enjoyment out of life.

Results from the logistic regression analysis that adjusts for sociodemographic and clinical characteristics are presented in Table 2, with the CES-D score as the dichotomous dependent variable (0-3 vs. 4+), using pooled data from the US and England. The country variable in 
the first row of the table represents the depressive symptom indicator in English adults compared to US adults (reference group). Without adjusting for other covariates, the prevalence of significant depressive symptoms (4+) in England in older adults was 25\% higher than the US. In the fully adjusted model, predictors associated with significant depressive symptoms included the country indicator, oldest age group (compared to youngest), female gender, heart and lung disease, current smoking, and ADL and IADL limitations, whereas predictors associated with lower levels of depressive symptoms included higher levels of education, net worth, and higher levels of cognitive functioning.

\section{Discussion}

This study provides direct comparisons of levels of depressive symptoms in the United States and England in nationally representative samples of older white adults. We found that rates of significant depressive symptoms were significantly higher in England, even after controlling for sociodemographic and clinical characteristics. Furthermore, despite higher levels of chronic diseases in the US, English respondents reported greater levels of ADL/ IADL impairments, which may be one factor leading to higher levels of significant depressive symptoms in England.

Our study suggests that one possible explanation for fewer depressive symptoms in the US may be due to higher levels of education and net worth in the US as compared to England, as both more education and greater net worth were associated with lower levels of significant depressive symptoms. These findings are in accordance with prior research indicating that higher socioeconomic status and education are associated with less depressive symptoms in older adults.(13)

Some key contributors to lower levels of significant depressive symptoms in the US may be the lower levels of ADL/IADL limitations and a lower level of cognitive impairment in the US. Previous research has confirmed a relationship between depression and both ADLs(14) and IADLs.(15) Furthermore, while the relationship between depression and cognitive impairment is complex, research has demonstrated a bidirectional association between cognitive impairment and depression.(16) It is important to try to tease out how ADL/IADL limitations, cognitive impairment, and depression are related over time (e.g. does depression lead to actual or perceived limitations in functioning or memory, or vice versa).

Interestingly, alcohol consumption, which was significantly higher in England, was also associated with lower odds of significant depressive symptoms in models that did not include ADL and IADL limitations (data not shown). While patients who have both alcohol use disorders and depression tend to be more depressed, more treatment resistant, and are more likely to commit suicide, a recent study found that moderate alcohol use was associated with lower levels of depression than abstinence (17). Although the English clearly drank more alcohol, data were not available to determine the prevalence of moderate and problematic drinking in each country. It is therefore uncertain to what extent differences in significant depressive symptoms may have been due to varying patterns of alcohol consumption in England and the US. Therefore, while the relationships among alcohol use, ADL/IADL limitations, and depression are unknown, this may deserve more attention given the significant relationship between drinking and depression in models that did not control for ADL/IADL limitations, and its non-significant relationship after controlling for them.

Despite known associations with depression and chronic diseases, we found that lung and heart disease were associated with increased odds of depression, but stroke, diabetes, and hypertension were not. The finding that lung disease was positively associated with significant depressive symptoms could be explained in part by the significant relationship 
between smoking (current or former) and significant depressive symptoms, which has also been found in other studies.(18) Numerous studies have documented the relationship between depression and heart disease.(19) Lung disease and heart disease have also been shown to be associated with increased depression symptoms over time in a Dutch longitudinal aging study.(20) While our findings of patterns between chronic disease and depression were similar to other studies, they likely do not explain the differences between depression in the US and England.

Studies generally indicate higher levels of depression among older women, but also declines in depression with age. While in adjusted analyses, we found higher levels of depressive symptoms in the oldest age groups, we found lower levels of significant depressive symptoms in the oldest age groups in both the US and England once functional limitations and cognitive impairment status were controlled for. Recent evidence suggests that this might be attributable to less treatment in older adults with depression. More research could examine cross national differences in depression by both gender and age groups to determine if there are changing trends in both depression prevalence and treatment in late adulthood.

While this study has provided new findings on the prevalence of depressive symptoms among older adults in the US and England, there are some limitations. This study is a cross sectional comparison of health and demographic characteristics of survey respondents in the US and England. Therefore, it is uncertain whether specific characteristics led to more depression (e.g. is having lower income or education a cause or consequence of depression), or whether depression led to a particular health or socioeconomic outcome, or perhaps these relationships were mediated by some other factors (e.g. financial strain or employment status may influence the relationship between depression and income or education). In addition, the same net worth in each country could represent different purchasing power (e.g. goods and services may cost more in England compared to the US), which could also influence levels of depressive symptoms. Future research should examine which factors influence depression status in each country over time, particularly because there may be other factors that we could not capture that influence differential rates of depressive symptoms in each country, including social norms, stigma, geographic location (including latitude and sunlight exposure), seasonality, and physical activity.

Another limitation is our inability to measure history of clinical depression or treatment for depression in these two populations, which could potentially explain a "depression advantage" in the United States (e.g. better treatment leading to better depression outcomes). We know from prior research that rates of depression treatment during the 1990s were much lower in England (less than 15\%) (21) than in the US (almost 75\%) (22). However, rates of antidepressant treatment have been increasing worldwide particularly since the introduction of selective serotonin reuptake inhibitors (SSRIs). In the US and England, population rates of SSRI use doubled over the last ten to fifteen years $(23,24)$. More data are needed to directly compare trends over time of depressive symptoms and antidepressant treatment in England and the US, to better disentangle whether and to what extent potentially higher levels of depression in England are related to lower rates of treatment.

There remains some uncertainty in how to interpret the meaning of a higher prevalence of depressive symptoms in England compared to the US. While the present study provides an important improvement over prior studies by using the same measure (CES-D) and nationally representative populations of older adults, it is still an open question as to whether respondents interpret the question items similarly in both countries. The issue of potential differential response to question items is of particular importance for depression because it cannot be measured directly using a biomarker, and thus detection and diagnosis is indirect. 
Therefore, future cross-national research might benefit from using vignettes to try to tease out differences in the interpretation of self reported health measures such as depression (25).

In addition, while both the HRS and ELSA are nationally representative samples, differences between the two studies in overall response rates, and in methods for the recruitment of proxy respondents to answer for sample members, could have important implications for the comparison of depressive symptoms of those included in our analysis. However, proxy status is usually based on whether a respondent is capable of answering questions on their own (e.g. proxies may be used for respondents with cognitive impairment), and depression is not likely a reason for proxy use. Furthermore, a similar pattern of national difference can be seen with moderate (1-3) and significant (>3) depressive symptoms, which makes it less likely that the effect is simply due to those with many depressive symptoms having proxy interviews in the US. Finally, people with depressive symptoms are more likely to be survey non-respondents in a population-based survey.(26) Given that ELSA had a lower response rate that HRS, if some of the nonresponse in both surveys was due to depressive symptoms, had those participants responded, we might have found even higher levels of depressive symptoms in England, and a larger difference between the US and English depressive symptoms levels, so our findings might actually be biased towards the null.

Furthermore, about $70 \%$ of the HRS sample was interviewed by telephone and $30 \%$ inperson, while all ELSA interviews were in-person. If telephone administration of the CES-D is associated with systematically different pattern of responses compared to in-person administration, this could explain some of the HRS depression advantage that we found. However, research indicates that asking questions about mood over the telephone does not yield different results than when these questions are asked in person.(27) In addition, we conducted a sensitivity analysis (data not shown) to determine whether mode of administration (telephone vs. in person) influenced depressive symptom levels, and found that it $\operatorname{did}$ not $(\mathrm{OR}=1.00, \mathrm{CI}: 0.79,1.25, \mathrm{p}=0.98)$.

In addition, while we limited our sample to respondents of white race only, due to the low number of non-white ELSA respondents, we recognize that potential greater racial diversity in the US may contribute to its health outcomes. The non-white plurality in the US may be less representative of the US than the non-white majority in England, which could potentially explain some of the apparent cross-border sociodemographic or health differences.

A final caution is that while the CES-D measures depressive symptoms and has good psychometric properties for measuring depression in older adults, it is not a diagnostic instrument. Therefore, we cannot make direct statements about rates of clinical depression diagnosis in each country, but rather can make comparisons of symptom levels between them.

\section{Conclusions}

This study demonstrated higher levels of depressive symptoms among older adults in England compared to the US, despite more chronic disease in the US. It is possible that these differences are attributable to higher levels of education and wealth as well as lower levels of ADL/IADL disabilities and cognitive impairment in the US. Given the negative impact that depression has on the quality of life and functioning of older adults, as populations age worldwide, it will be particularly important to understand the dynamic factors that influence the prevalence of depression within and between nations. 


\section{Acknowledgments}

The National Institute on Aging (NIA) provided funding for the Health and Retirement Study (HRS) (U01 AG09740), data from which were used for this analysis. The HRS is performed at the Institute for Social Research, University of Michigan. ELSA is funded by the US National Institute on Aging (NIA) and a consortium of UK Government departments. Dr. Zivin was funded by an NIA Pilot Faculty Grant to the Michigan Center on the Demography of Aging (MiCDA), and the Department of Veterans Affairs, Health Services Research and Development Service, CD2 07-206-1. Dr. Langa was supported by a grant from the NIA (R01 AG 027010).

\section{References}

1. Ustun TB, Ayuso-Mateos JL, Chatterji S, et al. Global burden of depressive disorders in the year 2000. British Journal of Psychiatry. 2004; 184:386-392. [PubMed: 15123501]

2. Beekman AT, Penninx BW, Deeg DJ, et al. The impact of depression on the well-being, disability and use of services in older adults: a longitudinal perspective. Acta Psychiatrica Scandinavica. 2002; 105:20-27. [PubMed: 12086221]

3. Zivin K, Kales HC. Adherence to depression treatment in older adults: a narrative review. Drugs Aging. 2008; 25:559-571. [PubMed: 18582145]

4. Banks J, Marmot M, Oldfield Z, et al. Disease and disadvantage in the United States and in England. JAMA. 2006; 295:2037-2045. [PubMed: 16670412]

5. Han B. Depressive symptoms and self-rated health in community-dwelling older adults: a longitudinal study. Journal of the American Geriatrics Society. 2002; 50:1549-1556. [PubMed: 12383153]

6. McDougall FA, Kvaal K, Matthews FE, et al. Prevalence of depression in older people in England and Wales: the MRC CFA Study. Psychological Medicine. 2007; 37:1787-1795. [PubMed: 17407617]

7. Gallo JJ, Lebowitz BD. The epidemiology of common late-life mental disorders in the community: themes for the new century. Psychiatric Services. 1999; 50:1158-1166. [PubMed: 10478901]

8. Copeland JR, Gurland BJ, Dewey ME, et al. Is there more dementia, depression and neurosis in New York? A comparative study of the elderly in New York and London using the computer diagnosis AGECAT. British Journal of Psychiatry. 1987; 151:466-473. [PubMed: 3328991]

9. Juster FT, Suzman R. An Overview of the Health and Retirement Study. Journal of Human Resources. 1995; 30(Supplement):S7-S56.

10. Marmot, M.; Banks, J.; Blundell, R., et al., editors. Health, wealth, and lifestyles of the older population in England The 2002 English Longitudinal Study of Ageing. Institute for Fiscal Studies; London, UK: 2002.

11. Radloff L. The CES-D scale: a self-report depression scale for research in the general population. Applied Psychological Measurement. 1977; 1:385-401.

12. Steffick, DE. Documentation of Affective Functioning Measures in the Health and Retirement Study. University of Michigan; Ann Arbor, MI: 2000.

13. Koster A, Bosma H, Kempen GI, et al. Socioeconomic differences in incident depression in older adults: the role of psychosocial factors, physical health status, and behavioral factors. Journal of Psychosomatic Research. 2006; 61:619-627. [PubMed: 17084139]

14. Penninx BW, Leveille S, Ferrucci L, et al. Exploring the effect of depression on physical disability: longitudinal evidence from the established populations for epidemiologic studies of the elderly. American Journal of Public Health. 1999; 89:1346-1352. [PubMed: 10474551]

15. Bruce ML, McAvay GJ, Raue PJ, et al. Major depression in elderly home health care patients. American Journal of Psychiatry. 2002; 159:1367-1374. [PubMed: 12153830]

16. Steffens DC, Otey E, Alexopoulos GS, et al. Perspectives on depression, mild cognitive impairment, and cognitive decline. Archives of General Psychiatry. 2006; 63:130-138. [PubMed: 16461855]

17. Lang I, Wallace RB, Huppert FA, et al. Moderate alcohol consumption in older adults is associated with better cognition and well-being than abstinence. Age and Ageing. 2007; 36:256-261.

[PubMed: 17353234] 
18. Himelhoch S, Daumit G. To whom do psychiatrists offer smoking-cessation counseling? American Journal of Psychiatry. 2003; 160:2228-2230. [PubMed: 14638595]

19. Frasure-Smith N, Lesperance F. Reflections on depression as a cardiac risk factor. Psychosomatic Medicine. 2005; 67:S19-25. [PubMed: 15953794]

20. Bisschop MI, Kriegsman DM, Deeg DJ, et al. The longitudinal relation between chronic diseases and depression in older persons in the community: the Longitudinal Aging Study Amsterdam. Journal of Clinical Epidemiology. 2004; 57:187-194. [PubMed: 15125629]

21. Copeland JRM, Beekman ATF, Braam AW, et al. Depression among older people in Europe: the EURODEP studies. World Psychiatry. 2004; 3:45-49. [PubMed: 16633454]

22. Olfson M, Marcus SC, Druss B, et al. National trends in the outpatient treatment of depression. JAMA. 2002; 287:203-209. [PubMed: 11779262]

23. Olfson M, Marcus SC, Druss BG. Effects of food and drug administration warnings on antidepressant use in a national sample. Archives of General Psychiatry. 2008; 65:94-101. [PubMed: 18180433]

24. Morgan O, Griffiths C, Majeed A. Antidepressant prescribing and changes in antidepressant poisoning mortality and suicide in England, 1993-2004. Journal of Public Health. 2008; 30:60-68. [PubMed: 18239187]

25. Banks J, Kapteyn A, Smith J, et al. Work Disability is a Pain in the*****, Especially in England, The Netherlands, and the United States. RAND Labor and Population Working Paper Series. 2005

26. Sales AE, Plomondon ME, Magid DJ, et al. Assessing response bias from missing quality of life data: the Heckman method. Health and Quality of Life Outcomes. 2004; 2:49. [PubMed: 15373945]

27. Monahan P, Moser L, Zhao Q, et al. The Indiana university telephone-based assessment of neuropsychological status: A new method for large scale neuropsychological assessment. Journal of the International Neuropsychological Society. 2007; 13:799-806. [PubMed: 17697411] 
Table 1

Characteristics of the US and English Samples, Age 65+, 2002

\begin{tabular}{|c|c|c|c|}
\hline Variable & $\begin{array}{c}\text { US } \\
(\mathrm{N}=\mathbf{8 , 2 9 5})\end{array}$ & $\begin{array}{c}\text { England } \\
(\mathbf{N}=5,208)\end{array}$ & P-value * \\
\hline Age & & & $\mathrm{F}(2.00,3078.28)=5.39 ; 0.005$ \\
\hline $65-74$ & 50.7 & 54.2 & \\
\hline $75-84$ & 38.1 & 35.8 & \\
\hline$\geq 85$ & 11.2 & 10.0 & \\
\hline Gender & & & $\mathrm{F}(1.00,1709.00)=14.26 ;<0.001$ \\
\hline Male & 40.2 & 42.9 & \\
\hline Female & 58.8 & 57.1 & \\
\hline Education ${ }^{* *}$ & & & $\mathrm{~F}(1.79,3053.92)=51.49 ;<0.001$ \\
\hline Low & 61.2 & 70.7 & \\
\hline Middle & 18.9 & 10.8 & \\
\hline High & 19.8 & 18.6 & \\
\hline Net worth (2002 US Dollars) & & & $\mathrm{F}(1.88,3213.60)=4.33 ; 0.02$ \\
\hline$\leq \$ 83,000$ & 29.5 & 33.4 & \\
\hline$\$ 83,001-\$ 318,000$ & 34.6 & 33.2 & \\
\hline$>\$ 318,000$ & 35.9 & 33.4 & \\
\hline Chronic Conditions & & & $\mathrm{F}(1.00,1709.00)$ \\
\hline Stroke & 8.2 & 6.8 & $7.58 ; 0.006$ \\
\hline Diabetes & 16.4 & 9.0 & $141.32 ;<0.001$ \\
\hline Heart disease & 56.6 & 44.8 & $163.57 ;<0.001$ \\
\hline Hypertension & 31.0 & 18.6 & $7.64 ; 0.006$ \\
\hline Lung disease & 11.3 & 8.1 & $32.47 ;<0.001$ \\
\hline Cancer & 17.6 & 7.8 & $238.78 ;<0.001$ \\
\hline$\#$ of $\mathrm{ADLs}^{\dagger}$ impaired & & & $F(1.99,3400.54)=17.79$ \\
\hline 0 & 77.1 & 72.2 & $<0.001$ \\
\hline $1-3$ & 19.5 & 24.2 & \\
\hline $4-6$ & 3.4 & 3.6 & \\
\hline$\#$ of IADLs $\%$ impaired & & & $F(1.95,3336.81)=13.23$ \\
\hline 0 & 85.5 & 83.0 & $<0.001$ \\
\hline $1-3$ & 12.8 & 16.0 & \\
\hline $4-5$ & 1.3 & 1.0 & \\
\hline Cognitive Function $(0-24)$ & & & $F(1.96,3355.33)=125.13$ \\
\hline Tertile & & & $<0.001$ \\
\hline Low $(0-11)$ & 34.8 & 47.0 & \\
\hline Medium $(12-14)$ & 31.4 & 31.4 & \\
\hline High $(15-24)$ & 33.7 & 21.6 & \\
\hline Alcohol intake (days / week) & & & $\mathrm{F}(2.92$, \\
\hline 0 & 54.3 & 15.5 & $4993.49)=358.40 ;<0.001$ \\
\hline$<1$ & 15.1 & 33.5 & \\
\hline
\end{tabular}



$>1$ to 2
13.6
17.0
24.4
$>2$
26.6

Smoking Status

Never

42.8

Former

Current

Depressive Symptoms

Depressed

Everything is an effort

Restless sleep

Was happy

Felt lonely

Enjoyed life

Felt sad

No motivation

Depressive Symptoms

0

$1-3$

$4-8$
47.7

9.5

35.2

52.4

12.4

$23.6 \quad 27.1$

$\begin{array}{ll}26.8 & 40.9\end{array}$

$88.4 \quad 89.7$

$19.0 \quad 17.4$

$93.0 \quad 90.0$

$20.6 \quad 22.1$

$24.3 \quad 25.0$

$43.2 \quad 36.5$

$41.9 \quad 45.9$

$14.9 \quad 17.6$

$16.1 \quad 19.4$

$\mathrm{F}(1.91,3261.37)=32.09$;

$<0.001$

$\mathrm{F}(1.00,1709.00)$

$17.71 ;<0.001$

$14.51 ;<0.001$

209.65; $<0.001$

4.16; 0.04

3.70; 0.05

$31.59 ;<0.001$

3.13; 0.08

$0.69 ; 0.4$

$\mathrm{F}(1.98,3380.27)=20.92$;

$<0.001$

Values are weighted percentages or means derived using the HRS and ELSA respondent population weights to adjust for the complex sampling design of each survey. This table uses Pearson chi-square statistics, which are corrected for the survey design, and converted to F statistics, including degrees of freedom.

P-value of chi-square or t-test for a significant difference in proportion or mean between countries.

${ }^{\dagger}$ ADLs indicates Activities of Daily Living (eating, transferring, toileting, dressing, bathing, and walking across a room).

FIADLs indicates Instrumental Activities of Daily Living (preparing meals, grocery shopping, making phone calls, taking medications, managing money).

**

Education categories-US: Low- $\leq 12$ years of school; Mid- 13 to 15 years; High- $\geq 16$ years. England: Low- lower than "O-level" (typically 0 to 11 years of school); Mid- "O-level" to lower than "A-level" (typically 12 to 13 years); High- "A-level" or higher (typically > 13 years).

Categories based on Banks et al 2006.(4) 
Table 2

Relative odds of depressive symptoms in $2002^{*}$

\begin{tabular}{|c|c|c|}
\hline Variable & $\begin{array}{c}\text { Unadjusted OR } \\
\left(95 \% \text { CI, t-value }{ }^{* *} ; \text { p-value }\right)\end{array}$ & $\begin{array}{c}\text { Adjusted OR } \\
\text { (95\% CI, t-value; } p \text {-value) }\end{array}$ \\
\hline Country (US = Ref) & $1.22(1.09-1.37,3.38 ; 0.001)$ & $1.18(1.03-1.36,2.4 ; 0.02)$ \\
\hline $\begin{array}{l}\text { Age } \\
\begin{array}{l}65-74 \\
75-84 \\
\geq 85\end{array}\end{array}$ & $\begin{array}{c}\text { Ref. } \\
1.26(1.13-1.40,4.2 ;<0.001) \\
1.77(1.54-2.04,7.9 ;<0.001)\end{array}$ & $\begin{array}{c}\text { Ref. } \\
0.94(0.85-1.04,-1.2 ; 0.2) \\
0.80(0.69-0.93,-2.9 ; 0.004)\end{array}$ \\
\hline Female gender & $1.76(1.55-2.00,8.7 ;<0.001)$ & $1.64(1.42-1.89,6.8 ;<0.001$ \\
\hline $\begin{array}{l}\text { Education } \\
\text { Low } \\
\text { Middle } \\
\text { High }\end{array}$ & $\begin{array}{c}\text { Ref. } \\
.61(.52-.70,-6.7 ;<0.001) \\
.43(.37-.50,-11.1 ;<0.001)\end{array}$ & $\begin{array}{c}\text { Ref. } \\
0.78(0.66-0.92,-2.9 ; 0.02) \\
0.69(0.58-0.83,-4.0 ;<0.001\end{array}$ \\
\hline $\begin{array}{l}\text { Net Worth }(2002 \$ s) \\
\quad \ll 82,000 \\
82,001-262,000 \\
>262,000\end{array}$ & $\begin{array}{c}\text { Ref. } \\
.54(.47-.63,-7.8 ;<0.001) \\
.33(.29-.38,-16.1 ;<0.001)\end{array}$ & $\begin{array}{c}\text { Ref. } \\
0.78(0.65-0.92,-2.9,0.004) \\
0.61(0.53-0.71,-6.4 ;<0.001\end{array}$ \\
\hline $\begin{array}{l}\text { Cardiovascular Risks } \\
\text { Stroke } \\
\text { Diabetes } \\
\text { Hypertension } \\
\text { Heart disease } \\
\text { Lung disease } \\
\text { Cancer }\end{array}$ & $\begin{array}{c}1.77(1.48-2.11,6.4 ;<0.001) \\
1.35(1.17-1.55,4.2 ;<0.001) \\
1.28(1.13-1.44,4.0 ;<0.001) \\
1.74(1.54-1.97,8.9 ;<0.001) \\
2.14(1.85-2.48,10.2 ;<0.001) \\
1.06(.92-1.22, .8 ; 0.4)\end{array}$ & $\begin{array}{c}0.97(0.82-1.15,-.4 ; 0.7) \\
1.02(0.85-1.21, .2 ; 0.9) \\
1.02(0.90-1.17, .4 ; 0.7) \\
1.35(1.21-1.51,5.3 ;<0.001) \\
1.44(1.23-1.68,4.5 ;<0.001) \\
1.04(0.89-1.23, .5 ; 0.6)\end{array}$ \\
\hline $\begin{array}{l}\text { Cognitive Impairment } \\
0-11 \\
12-14 \\
15-24\end{array}$ & $\begin{array}{c}\text { Ref. } \\
.66(.59-.73,-8.0 ;<0.001) \\
.46(.39-.53,-10.5 ;<0.001)\end{array}$ & $\begin{array}{c}\text { Ref. } \\
0.91(0.81-1.01,-1.7 ; 0.08) \\
0.72(0.60-0.85,-3.8 ;<0.001)\end{array}$ \\
\hline $\begin{array}{l}\text { Alcohol } \\
0 \\
<1 \\
>1 \text { to } 2 \\
>2\end{array}$ & $\begin{array}{c}\text { Ref. } \\
.71(.61-.82,-4.5 ;<0.001) \\
.55(.47-.64,-7.5 ;<0.001) \\
.48(.41-.57,-8.4 ;<0.001)\end{array}$ & $\begin{array}{c}\text { Ref. } \\
0.84(0.71-.99,-2.04 ; 0.04) \\
0.84(0.71-1.0,-1.95 ; 0.05) \\
0.78(0.65-.95,-2.5 ; 0.01)\end{array}$ \\
\hline $\begin{array}{l}\text { Smoking Status } \\
\text { Never } \\
\text { Former } \\
\text { Current }\end{array}$ & $\begin{array}{c}\text { Ref. } \\
1.07(.98-1.17,1.6 ; 0.1) \\
1.62(1.38-1.89,6.0 ;<0.001)\end{array}$ & $\begin{array}{c}\text { Ref. } \\
1.19(1.07-1.32,3.3 ; 0.001) \\
1.54(1.31-1.80,5.3 ;<0.001)\end{array}$ \\
\hline $\begin{array}{l}\text { \# ADLs } \\
0 \\
1-3 \\
4-6\end{array}$ & $\begin{array}{c}\text { Ref. } \\
3.56(3.19-3.97,22.9 ;<0.001) \\
7.73(6.28-9.51,19.4 ;<0.001)\end{array}$ & $\begin{array}{c}\text { Ref. } \\
2.25(1.98-2.56,12.6 ;<0.001) \\
3.35(2.65-4.23,10.2 ;<0.001)\end{array}$ \\
\hline $\begin{array}{l}\text { \# IADLs } \\
0 \\
1-3 \\
4-5\end{array}$ & $\begin{array}{c}\text { Ref. } \\
4.14(3.75-4.58,27.8 ;<0.001) \\
5.43(3.56-8.27,7.9 ;<0.001\end{array}$ & $\begin{array}{c}\text { Ref. } \\
1.93(1.68-2.22,9.1 ;<0.001) \\
2.12(1.37-3.27,3.4 ; 0.001)\end{array}$ \\
\hline
\end{tabular}

Adjusted models adjust for all variables in the table.

Models use t-tests, which approximate the Z-distribution for a large sample size 